\section{A) Check for updates}

Cite this: Dalton Trans., 2020, 49, 17674

Received 11th August 2020,

Accepted 1st November 2020

DOI: $10.1039 / \mathrm{d} 0 \mathrm{dt} 02818 \mathrm{e}$

rsc.li/dalton

\title{
Modular O- vs. N-coordination of pyridylidene amide ligands to iron determines activity in alcohol oxidation catalysis $\uparrow$
}

\begin{abstract}
Dide G. A. Verhoeven and Martin Albrecht (iD) *
A family of polydentate pyridine-substituted pyridylidene amide (PYA) complexes bound to iron(II) was developed. The variation of the coordination set from NN-bidentate PYA to tridentate pincer-type pyPYA 2 systems ( pyPYA $2=2,6$-bis(PYA)pyridine) had a large influence on the binding mode to iron(I), including a change from the $\mathrm{N}$ - to rare $\mathrm{O}$-coordination of the PYA site and a concomitant shift of the predominant ligand resonance structure. These binding mode variations invoke changes in the reactivity of the complexes, which were probed in the peroxide-mediated oxidation of 1-phenylethanol to acetophenone. A comparison with uncomplexed $\mathrm{FeCl}_{2}$ indicated that bidentate $\mathrm{NN}$ coordination is unstable and presumably leads to the dissociation of $\mathrm{FeCl}_{2}$. In contrast, the tridentate ligand binding is robust. Remarkably, the tridentate PYA pincer coordination inhibits catalytic activity in the NNN binding mode, while the ONO coordination greatly enhances catalytic performance. Under optimized conditions, the bis-ligated ONO pincer iron complex $\left[\mathrm{Fe}\left(\mathrm{pyPYA}_{2}\right)_{2}\right]\left[2 \mathrm{PF}_{6}\right]$ reaches full conversion within one hour $(0.5 \mathrm{~mol} \%$ catalyst loading) and under dilute conditions turnover numbers over 20000 (0.005 mol\% catalyst loading).
\end{abstract}

\section{Introduction}

Neutral N-donor ligands are ubiquitous in homogeneous catalysis, including imines, amines, pyridines, and as a more recent addition, pyridylidene amide (PYA) ligands. ${ }^{1,2}$ This latter scaffold has been emerging as a promising class of ligands with strong $\sigma$-donating properties. Analysis of PYA's $\sigma$-donor strength has been shown to resemble that of common C-coordinating N-heterocyclic carbene (NHC) ligands, making them unique nitrogen-based ligands for homogeneous catalysis. ${ }^{2-5}$ PYAs consist of a deprotonated amide core bound to an $N$-alkylated pyridinium ring (Scheme 1). These ligands constitute an overall neutral donor system which is comprised of multiple limiting resonance structures including the neutral imine structure $\mathbf{A}$ and zwitterionic structures $\mathbf{B}$ and $\mathbf{C .}^{2,6}$ Whereas evidence for resonance structures $\mathbf{A}$ and $\mathbf{B}$ have been obtained, for example from crystallographic and NMR data, ${ }^{6-9}$ structure $\mathbf{C}$ has solely been proposed. ${ }^{10}$ As a direct result of these multiple resonance structures, the ligand is electronically flexible in its donor properties to metal centers: it can

Departement für Chemie und Biochemie, Universität Bern, Freiestrasse 3, CH-3012 Bern, Switzerland.E-mail: martin.albrecht@dcb.unibe.ch

$\dagger$ Electronic supplementary information (ESI) available: Spectroscopic and electrochemical data, catalytic and crystallographic details. CCDC 2001555-2001558. For ESI and crystallographic data in CIF or other electronic format see DOI: 10.1039/D0DT02818E alter between L-type bonding through resonance form $\mathbf{A}$ and $\mathrm{X}$-type metal coordination through resonance form $\mathbf{B}$, and therefore adapt to the influence and requirements of the metal center. Pyridine substituted PYA ligands (Scheme 1, $\mathrm{R}^{\prime}=2$-pyridine) are related to the more common pyridine-bis-carboxamide ligands. ${ }^{11}$ However, the formally neutral nature of the PYA ligands and their electronically flexible behavior impart unique properties when compared to the more frequently used dianionic and electronically static pyridine bis-carboxamide systems. For example, the CN-coordinating PYA ligand in the complex $\left[\operatorname{Ir}(\mathrm{PhPYA})\left(\mathrm{Cp}^{*}\right) \mathrm{L}\right]$ adapts its binding mode from a larger contribution of resonance form $\mathbf{A}$ when $\mathrm{L}=\mathrm{Cl}$ to predominantly form $\mathbf{B}$ in the solvento complex $(\mathrm{L}=\mathrm{MeCN})$ to compensate for the differently charged ligands L (Fig. 1). ${ }^{12}$ Owing to this electronic flexibility, the PYA ligands can stabilize multiple oxidation states on a metal center and their use has brought attractive results to precious metal complexes in homogeneous catalysis (Fig. 2), such as $\mathrm{Pd}$ (pyPYA) $\mathrm{Cl}_{2}$ for cross coupling reactions, ${ }^{2} \operatorname{Ir}(\mathrm{PhPYA})\left(\mathrm{Cp}^{*}\right) \mathrm{Cl}$ for water oxidation ${ }^{13}$ and $[\mathrm{Ru}(\mathrm{bisPYA})(p$-cym $) \mathrm{Cl}] \mathrm{PF}_{6}$ for olefin oxidation. ${ }^{14}$

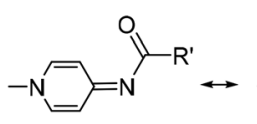

A

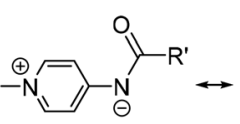

B

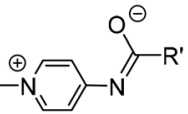

C
Scheme 1 Resonance structures of a generalized PYA ligand. 


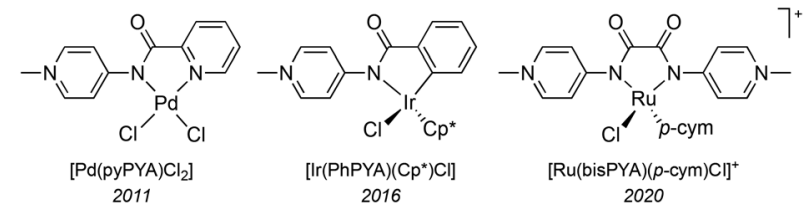

Fig. 1 Selected literature precedents of PYA complexes: $\left[\mathrm{Pd}\left(\right.\right.$ pyPYA $\left.{ }^{R}\right)$ $\left.\mathrm{Cl}_{2}\right]\left(\mathrm{R}=\mathrm{Me}\right.$, benzyl), $\left.\operatorname{lr}(\mathrm{PhPYA})\left(\mathrm{C} \mathrm{p}^{*}\right) \mathrm{L}\right](\mathrm{L}=\mathrm{Cl}, \mathrm{MeCN})$, and $[\mathrm{Ru}($ bisPYA)$(p$-cym)Cl]PF .

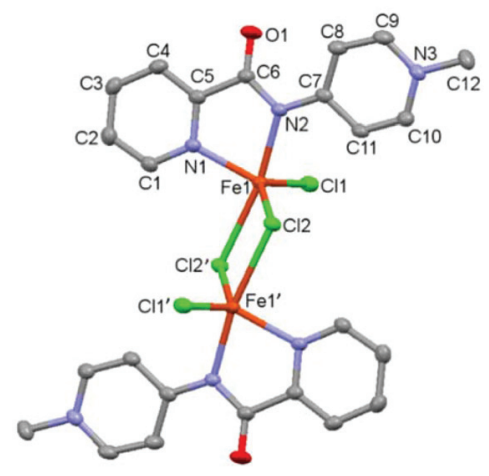

Fig. 2 ORTEP representation of complex 2 (50\% probability ellipsoids: hydrogen atoms omitted for clarity). Selected bond distances in Å: Fe1N1: 2.1142(16), Fe1-N2: 2.1639(15), O1-C6: 1.229(2), N2-C6: 1.361(2), N2-C7: 1.378(2), N3-C9: 1.346(3), N3-C10: 1.354(2), N3-C12: 1.476(2), C5-C6: 1.511(3), C7-C8: 1.418(3), C7-C11: 1.411(3), C8-C9: 1.362(3), C10-C11: 1.365(3). Bond angle N1-Fe1-N2: 78.19(6) ${ }^{\circ}$.

Their N-coordination and donor flexibility make PYA ligands particularly attractive for hard and Earth-abundant first row transition metals, offering the opportunity to stabilize catalytic intermediates of different oxidation states. For example, multidentate nitrogen donor ligands bound to iron have provided attractive catalytic performance for $\mathrm{C}-\mathrm{H}$ oxidation reactions, ${ }^{15-17}$ olefin polymerization, ${ }^{18-20}$ and olefin oxidation. ${ }^{21}$ Considering these facts, it is remarkable that no PYA ligand has been coordinated to iron. Here, we report the coordination of polydentate PYA ligands to iron and their catalytic activity in oxidation catalysis. Changes in the ligand structure greatly impact the stability, coordination mode, and catalytic activity of the complexes and reveal evidence of the rare O-coordination of these PYA ligands.

\section{Results and discussion}

The potentially NN-bidentate coordinating pyridine-substituted PYA ligand $\left(\mathbf{1}^{\mathbf{I}}\right)$ was synthesized following previously reported protocols. ${ }^{2,9}$ Subsequent anion exchange with $\mathrm{NH}_{4} \mathrm{PF}_{6}$ in water yielded $\mathbf{1}^{\mathbf{P F} 6}$ (Scheme 2). The coordination of $\mathbf{1}^{\mathbf{P F 6}}$ to $\mathrm{FeCl}_{2}$ was accomplished by a stepwise process including first ligand deprotonation with a stoichiometric amount of LiHMDS as a strong non-nucleophilic base, followed by the addition of the metal precursor, which afforded complex 2 after precipitation with $\mathrm{Et}_{2} \mathrm{O}$. ${ }^{1} \mathrm{H}$ NMR analysis in $\mathrm{CD}_{3} \mathrm{CN}$ showed signals ranging from -50 to

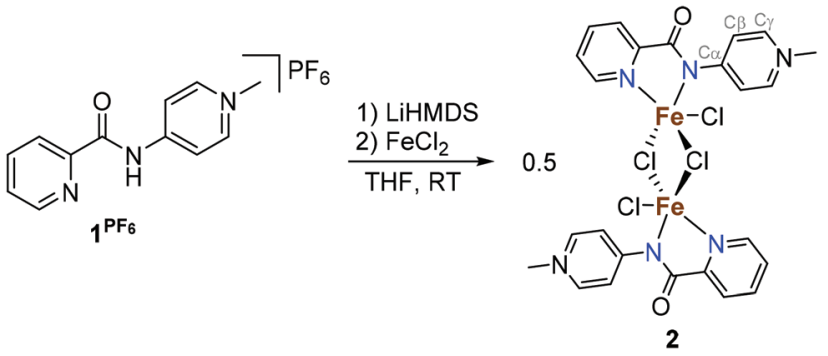

Scheme 2 Synthesis of 2. $C_{\alpha}, C_{\beta}$ and $C_{\gamma}$ are defined in 2.

+73 ppm which are consistent with a paramagnetic compound (Fig. S5†). Magnetic susceptibility measurements performed using the Evans method gave a $\mu_{\text {eff }}$ of 4.8 for each iron unit indicating a high spin configuration of the $\mathrm{d}^{6}$ metal with $S=2 .^{22}$ In line with this finding, no signal was obtained in the X-band EPR spectrum (solid state and DMSO solution at RT and $77 \mathrm{~K}$ ). Cyclic voltammetry measurements in $\mathrm{MeCN}$ indicate the presence of two oxidative waves at $E_{\mathrm{pa}}=1.16$ and $2.01 \mathrm{~V}$ (vs. $\mathrm{Fc}^{+} / \mathrm{Fc}$, Fig. S16 and Table $\mathrm{S} 1 \dagger)$. Both oxidations were irreversible under the employed conditions, presumably due to the loss of a chloride ligand or complex decomposition. Additionally, stability tests by ${ }^{1} \mathrm{H}$ NMR spectroscopy indicated that the complex is air and moisture sensitive. Exposure of the complex to air directly results in the formation of the free ligand and the disappearance of the paramagnetic signals, both in the solid state and in solution.

Single crystals suitable for X-ray diffraction analysis were grown by the slow evaporation of a MeCN solution under inert conditions, and identified complex 2 as a dimeric structure (Fig. 2). ${ }^{23}$ Each asymmetric unit in the crystal consists of an iron center bound to two nitrogen atoms of the bidentate chelating pyridyl-PYA ligand, and a terminal as well as two bridging chlorido ligands. The $\mathrm{C}-\mathrm{C}$ bond lengths in the PYA pyridyl ring show bond length alteration, since the $\mathrm{C}_{\alpha}-\mathrm{C}_{\beta}$ bond is elongated compared to the $\mathrm{C}_{\beta}-\mathrm{C}_{\gamma}$ bond with an average difference of $0.051 \AA$ (Table $\mathrm{S} 4 \dagger$ ), which indicates some contribution of the diene resonance form $\mathbf{A}$ ( $c f$. Scheme 1).

In an attempt to break this dimer, we explored the addition of stoichiometric amounts of co-ligands, such as 1,2-bis(diphenylphosphino)ethane (dppe), 2,2'-bipyridine (bpy) or ethylenediamine (en), though no reaction was observed. However, stirring the dimer with stoichiometric quantities of $\mathrm{NEt}_{4} \mathrm{I}$ in $\mathrm{THF} /$ pyridine in a $4: 1$ ratio for several hours and the subsequent crystallization of the mixture revealed the formation of small quantities of monomeric complex 3 as a highly air-sensitive complex. $^{24}$ The molecular structure of $\mathbf{3}$ was determined by $\mathrm{X}$-ray diffraction analysis and consists of an octahedral $\mathrm{Fe}^{\mathrm{II}}$ center bound to chloride, three pyridine ligands, and the $\kappa^{2}(\mathrm{~N}, \mathrm{O})$ coordinated PYA ligand, implying a rearrangement of the $\mathrm{N}$-binding mode of the amide moiety to the untypical O-coordination mode (Fig. 3). ${ }^{25} \mathrm{~N}$-coordination of the PYA site instead of $\mathrm{O}$-coordination induces significant changes in the amide unit, with elongation of the C6-O1 bond in 3 compared to $2(1.272(3)$ vs. $1.229(2) \AA)$ along with a shortening of C6-N2 


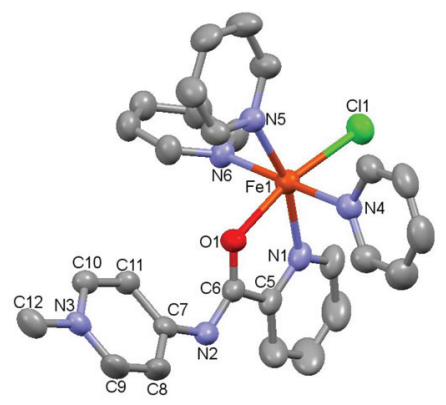

Fig. 3 ORTEP representation of complex 3 (50\% probability ellipsoids; hydrogen atoms and iodide counterions omitted for clarity). Selected bond distances in $\AA$ : Fe1-Cl1: 2.3914(8), Fe1-O1: 2.0927(18), Fe1-N1: 2.189(2), C5-C6: 1.504(4), C7-C8: 1.400(4), C7-C11: 1.405(4), O1-C6: 1.272(3), C8-C9: 1.355(4), C10-C11: 1.354(4), N1-C5: 1.344(4), N2-C6: 1.308(3), N2-C7: 1.377(4), N3-C9: 1.348(4), N3-C10: 1.344(3), N3-C12: 1.474(4). Bond angle O1-Fe1-N1: 75.74(8) ${ }^{\circ}$.

bond from 1.378(2) $\AA$ in 2 to 1.308(3) $\AA$ in 3. These bond length changes reflect a more pronounced contribution of the ${ }^{+} \mathrm{N}=\mathrm{C}-\mathrm{O}^{-}$amide resonance form and indicate the increased relevance of resonance structure $\mathbf{C}$, featuring the formal negative charge located at the oxygen atom ( $c f$. Scheme 1). Moreover, complex 3 reveals partial double bond localization in the pyridyl heterocycle. The $\mathrm{C}_{\alpha}-\mathrm{C}_{\beta}$ bonds are elongated compared to the $\mathrm{C}_{\beta}-\mathrm{C}_{\gamma}$ bonds with an average difference of $0.048 \AA$ (Table S5†). This difference is similar to that observed in complex 2 and suggests that either $\mathrm{C}-\mathrm{C}$ bond length analysis is not a useful probe to distinguish the contributions of different resonance structures or that other resonance structures contribute as well; for example, a diene-type structure with the positive and negative charge located on the amide unit $\left(=\mathrm{N}^{+}=\mathrm{C}(\mathrm{R})-\mathrm{O}^{-}\right)$.

\section{Pincer PYA ligands}

To increase the stability of the iron PYA complexes, the chelation of the ligand was extended to a tridentate coordinating pincer-type system comprising a central pyridine unit with two PYA arms derived either from para or ortho aminopyridine $\left(4^{\mathrm{PF} 6}\right.$ and $5^{\mathrm{PF} 6}$, Scheme 3). ${ }^{26-28}$ Complexation of $4^{\mathrm{PF6}}$ was performed by the deprotonation of the ligand precursor in the presence of $\mathrm{Na}_{2} \mathrm{CO}_{3}$ in DMF, followed by filtration into a DMF solution of $\mathrm{FeCl}_{2}$, which induced the precipitation of complex 6 (Scheme 3). The neutral iron(II) complex is paramagnetic as evident from the ${ }^{1} \mathrm{H}$ NMR spectra with shifts in the range of 0 to $73 \mathrm{ppm}$ and from the magnetic susceptibility of $\mu_{\text {eff }}=4.9$ (Evans method), consistent with an $S=2$ ground state (Fig. S8†). Complex 6 shows two quasi reversible and presumably metal-centered redox processes in cyclic voltammetry (Fig. $\mathrm{S} 17 \dagger)^{29}$ at $E_{1 / 2}=-0.46$ and $-0.31 \mathrm{~V}$ (vs. $\mathrm{Fc}^{+} / \mathrm{Fc}$ in DMF). Single crystals suitable for crystallographic analysis were grown by slow vapor diffusion of $\mathrm{Et}_{2} \mathrm{O}$ into a concentrated solution of the complex in DMSO. The molecular structure of 6 reveals an iron center bound to a $\kappa^{3}(\mathrm{~N}, \mathrm{~N}, \mathrm{~N})$-coordinated PYA pincer ligand and two chloride ligands, resulting in a five-coordinate trigonal bipyramidal geometry.

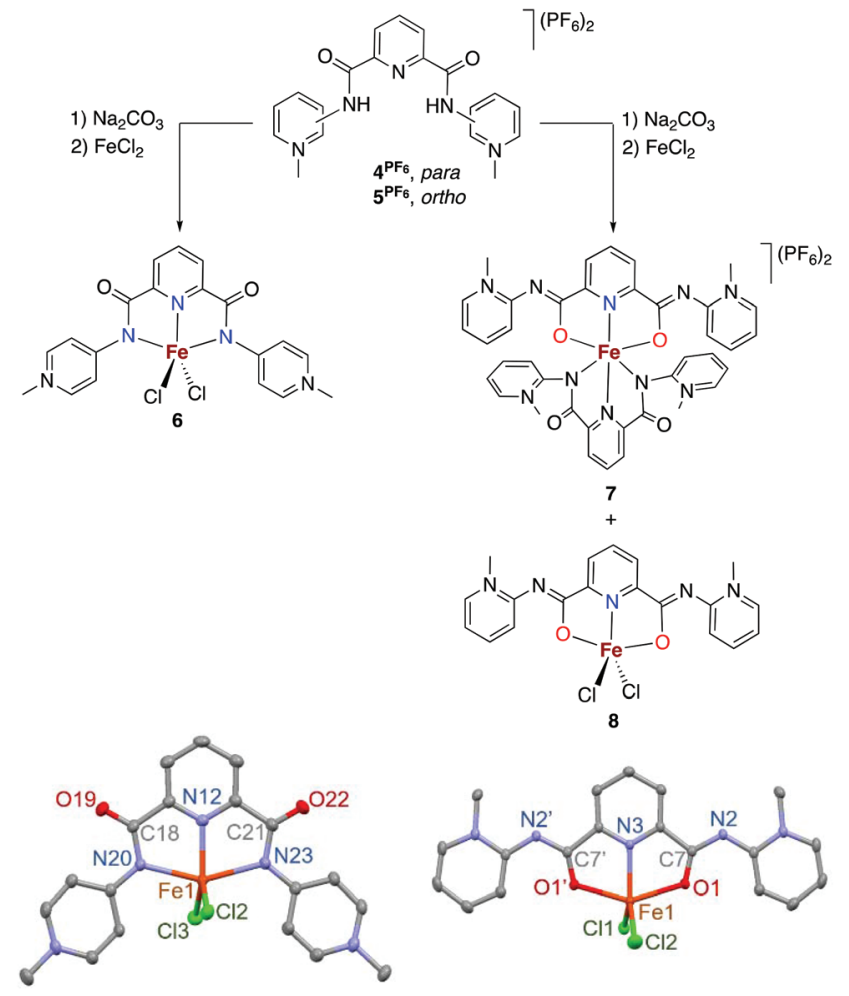

Scheme 3 Synthesis (top) and ORTEP plots (bottom) of complexes 6-8 and ORTEP plots of 6 and 8 shown at 50\% thermal ellipsoids, hydrogen atoms and co-crystallized DMSO molecules are omitted for clarity. Selected bond distances in Å: 6: Fe1-N12: 2.0696(14), Fe1-N20: 2.2476(14), Fe1-N23: 2.2559(14), O19-C18: 1.231(2), O22-C21: 1.233(2), N20-C18: 1.363(2), N23-C21: 1.357(2). 8: Fe1-N3: 2.109(2), Fe1-O1: 2.1797(12), O1-C7: 1.259(2), N2-C7: 1.329(2).

In contrast, the complexation of the ortho-PYA pincer system $5^{\mathbf{P F} 6}$ by the same procedure resulted in the precipitation of bis-pincer iron(II) complex 7. The purple powder is only sparingly soluble in common solvents, including MeCN and DMF, hampering its recrystallization. $\mathrm{A}^{1} \mathrm{H}$ NMR analysis in DMSO- $\mathrm{d}_{6}$ showed that 7 is paramagnetic with shifts ranging from 0 to $70 \mathrm{ppm}$ and that the complex has only limited stability in this solvent (Fig. S9†) ${ }^{30}$ A CV analysis of 7 reveals oxidation at higher potential compared to that of 6 with $E_{1 / 2}=$ -0.25 and $-0.05 \mathrm{~V}$ ( $v s . \mathrm{Fc}^{+} / \mathrm{Fc}$ in $\left.\mathrm{MeCN}\right)$. The formation of 7 was further supported by HRMS and elemental analysis. Notably, the DMF supernatant from the synthesis of 7 contained traces of a different complex, which crystallized as the mono-pincer complex 8 (Fig. S19†). This complex might be an intermediate in the formation of the bis-pincer complex, which is also the favored product in relation to $\mathrm{Co}^{\mathrm{II}}$ and $\mathrm{Ni}^{\mathrm{II}}$ chemistry. ${ }^{28}$ In analogy to these cobalt and nickel complexes, the ortho-PYA pincer ligand in the iron(II) complex 8 features a rare ONO-tridentate bonding mode, presumably because of a combination of effects including steric shielding of the amide nitrogen by the ortho-substitution, electronic factors associated with the relatively weak donor properties of $\mathrm{N}$-coordinating ortho PYA ligands, as well as the hardness of the $3 \mathrm{~d}$ metal 
center. ${ }^{31}$ While the attempts to crystallize complex 7 have failed so far, we speculate that at least one of the pyPYA ligands is bound in the ONO-coordination mode based on the structure of $\mathbf{8}$ and the analogy to other first-row transition metal complexes with this ligand. ${ }^{28}$

Complexes $\mathbf{6}$ and 8 are structurally related to anionic pyridine bis-carboxamide complexes such as $\left[\mathrm{Fe}^{\mathrm{III}}(2,6-\mathrm{bis}(N-\right.$ phenylcarbamoyl)pyridine) $\left.)_{2}\right]\left[\mathrm{NEt}_{4}\right]^{32}{ }^{32}$ Generally, these doubly anionic ligands coordinate through the nitrogen atoms, but steric congestion indicated that O-coordination is also feasible. ${ }^{33,34}$ Bond length analyses in the N-bound para-PYA unit in 6 revealed an average difference of $0.052 \AA$ between $\mathrm{C}_{\alpha}-\mathrm{C}_{\beta}$ and $\mathrm{C}_{\beta}-\mathrm{C}_{\gamma}$, suggesting similar double bond localization as noted in complexes 2 and 3 (Table S6†). Remarkably, this bond length alteration is just slightly less pronounced in the O-bound ortho-PYA heterocycle of complex 8 ( $\Delta d \quad 0.034 \AA$; Table $\mathrm{S} 7 \dagger)$. In addition, the amide $\mathrm{C}=\mathrm{O}$ bond is only moderately elongated at 1.259(2) $\AA$ (cf. 1.232(3) in 6 and 1.272(3) in $3)$, while the exocylic $\mathrm{C}_{\mathrm{PYA}_{\mathrm{A}}}-\mathrm{N}_{\text {amid }}$ bond is shortened to 1.353(2) $\AA$. Together with the contraction of the adjacent $\mathrm{C}-\mathrm{N}$ bond to $1.329(2) \AA$ ( $c f .1 .360(5) \AA$ in 6), these data indicate relevant contributions of resonance structures $\mathbf{A}$ and $\mathbf{C}$ for complex $\mathbf{8}$.

\section{Alcohol oxidation catalysis}

We have used catalytic alcohol oxidation as a probe to evaluate the effect of the different ligand coordination modes (Table 1 and Table S2 $\dagger$ ). Initially, the reactivity of complex 2 was explored in the catalytic oxidation of 1-phenyl ethanol to acetophenone as a model reaction. ${ }^{35-39}$ Under standard conditions, viz. $0.5 \mathrm{~mol} \%$ of the dimeric iron complex 2, $1.5 \mathrm{eq}$. $t$-butyl hydroperoxide (TBHP), $50{ }^{\circ} \mathrm{C}$ in $\mathrm{MeCN}$, complex 2 cleanly converted the alcohol to acetophenone and reached full conversion after $24 \mathrm{~h}$, slightly slower than when using only $\mathrm{FeCl}_{2}$ as the catalyst precursor (Table 1, entries 1,2). Substituting TBHP by $30 \%$ aq. $\mathrm{H}_{2} \mathrm{O}_{2}$ suppressed product formation, which is likely caused by the sensitivity of complex 2 towards water. Under mild conditions ( $0.1 \mathrm{~mol} \%$ catalyst at $25{ }^{\circ} \mathrm{C}$ ), complex 2 and $\mathrm{FeCl}_{2}$ have comparable reactivity, reaching a product yield of $18 \pm 1 \%$ after 2 days (entries 6, 7; Fig. S18†). This similar activity suggests the formation of an identical catalytically active species when starting from 2 or $\mathrm{FeCl}_{2}$, i.e., catalyst activation of complex 2 presumably involves ligand dissociation and the release of $\mathrm{FeCl}_{2}$ as an active entity.

In contrast, the pincer type complexes 6 and 7 showed diverging reactivities. Complex 6 with an N,N,N-coordinated PYA pincer ligand only yielded $48 \%$ product after 24 h (entry 3 , cf. $>80 \%$ for $\mathrm{FeCl}_{2}$ and complex 2). However, complex 7 reached full conversion after $24 \mathrm{~h}$ and resulted in a higher yield at $2 \mathrm{~h}$ when compared to $2(47 \%$ vs. $33 \%$, entry 4$) .^{40}$ Even at a catalyst loading of $0.5 \mathrm{~mol} \%, 7$ accomplishes full conversion after $24 \mathrm{~h}$ (entry 5). Comparing the performance at lower catalyst loading and milder temperatures, viz. $0.1 \mathrm{~mol} \%$ and $25{ }^{\circ} \mathrm{C}$, demonstrates the distinct activity of complex 7. ${ }^{41}$ Under these conditions, $\mathrm{FeCl}_{2}$ and complex 2 reached 17 and 18\% yield, respectively, after 2 days (entries 6,
Table 1 Catalytic alcohol oxidation of 1-phenylethanol to acetophenone $^{a}$

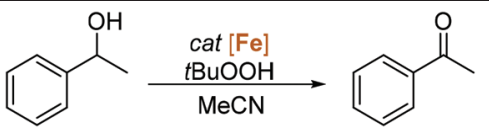

\begin{tabular}{llllll}
\hline Entry & {$[\mathrm{Fe}]$} & $\begin{array}{l}\text { Fe loading } \\
(\mathrm{mol} \%)\end{array}$ & $\begin{array}{l}\text { Temp. } \\
\left({ }^{\circ} \mathrm{C}\right)\end{array}$ & \multicolumn{2}{l}{ Conversion (time) } \\
\hline 1 & $\mathrm{FeCl}_{2}$ & 1 & 50 & $45(2 \mathrm{~h})$ & $81(24 \mathrm{~h})$ \\
$2^{b}$ & 2 & 1 & 50 & $33(2 \mathrm{~h})$ & $>99(24 \mathrm{~h})$ \\
3 & $\mathbf{6}$ & 1 & 50 & $11(2 \mathrm{~h})$ & $48(24 \mathrm{~h})$ \\
4 & 7 & 1 & 50 & $47(2 \mathrm{~h})$ & $>99(24 \mathrm{~h})$ \\
5 & 7 & 0.5 & 50 & $42(2 \mathrm{~h})$ & $>99(24 \mathrm{~h})$ \\
6 & $\mathrm{FeCl}_{2}$ & 0.1 & 25 & & $17(2 \mathrm{~d})$ \\
$7^{b}$ & 2 & 0.1 & 25 & & $18(2 \mathrm{~d})$ \\
8 & $\mathbf{6}$ & 0.1 & 25 & & $6(2 \mathrm{~d})$ \\
9 & 7 & 0.1 & 25 & & $67(2 \mathrm{~d})$ \\
10 & 7 & 0.05 & 25 & & $58(2 \mathrm{~d})$ \\
$11^{c}$ & $7+\mathrm{Ph}_{2} \mathrm{NH}$ & 0.5 & 50 & $<3(1 \mathrm{~h})$ & $<3(24 \mathrm{~h})$ \\
$12^{d}$ & 7 & 0.5 & 80 & $98(1 \mathrm{~h})$ & \\
$13^{d}$ & $\mathrm{FeCl}_{2}$ & 1 & 80 & $50(1 \mathrm{~h})$ & \\
14 & $\mathbf{5}^{\mathbf{P F 6}}$ & 0 & 80 & $1(1 \mathrm{~h})$ & \\
15 & none & 0 & 80 & $<5(1 \mathrm{~h})$ & \\
$16^{e}$ & 7 & 0.005 & 80 & $92(24 \mathrm{~h})$ & $98(48 \mathrm{~h})$
\end{tabular}

${ }^{a}$ General conditions: iron complex $(7 \mu \mathrm{mol}, 1 \mathrm{~mol} \%)$, MeCN $(0.5 \mathrm{~mL})$, 1-phenylethanol $(0.7 \mathrm{mmol})$, TBHP (1.05 mmol, $5 \mathrm{M}$ in decane), $\mathrm{Ar}$ atmosphere, yields determined by ${ }^{1} \mathrm{H}$ NMR spectroscopy (Fig. S10 $\dagger$ ); quantities of substrate and catalyst adjusted for runs with lower catalyst loading, see experimental for details. ${ }^{b} 0.5 \mathrm{~mol} \%$ of the dimeric complex was used to keep [Fe] at $1 \mathrm{~mol} \%{ }^{c} 2$ equiv. diphenylamine added at the start of the reaction. ${ }^{d}$ TBHP was added in two portions, 1 equiv. at $t=0$ and 1 equiv. at $t=30 \mathrm{~min} .{ }^{e} 1.5$ equiv. TBHP added in two portions, 1 equiv. at $t=0$ and 0.5 equiv. at $t=20 \mathrm{~h}$.

7) and complex 6 achieved a modest 6\% (entry 8); yet, complex 7 accelerated product formation and afforded an appreciable $67 \%$ yield within the same time span (entry 9). Conversions of almost $60 \%$ were accomplished at even a lower catalyst loading of 0.05 mol\% (entry 10), ${ }^{42}$ corresponding to turnover numbers (TONs) of 1960. These runs also indicate a considerable rate enhancement compared to $\mathrm{FeCl}_{2}$.

These catalytic experiments suggest that the bidentate ligand system is not binding strong enough and leads to catalytic activity that is commensurate with ligand dissociation ( $c f$. identical activity of $\mathrm{FeCl}_{2}$ ). Unlike the bidentate system, the tridentate pincer-type coordination has a much more profound impact on complex integrity and catalytic activity (see also Table $\mathrm{S} 2 \uparrow$ for various control experiments). The NNN coordination mode of the para-PYA pincer ligand in 6 inhibits catalytic activity significantly, suggesting a tight bonding of the Fe center within the pincer ligand scaffold, while the bis-pincer coordination pattern in complex 7 imparts a substantially enhanced catalytic performance, presumably after the loss of one of the pincer ligands. Even though steric effects cannot be excluded, we surmise that the electronic implication of the PYA resonance structure $\mathbf{C}$ with partially anionic oxygen donors beneficially influences the electronic configuration at the iron center to stabilize critical transition states during 
metal oxidation. In particular, higher oxidation species generated in the reaction of TBHP with the $\mathrm{Fe}^{\mathrm{II}}$ complex are expected to be better stabilized by the ONO-pincer coordination mode due to the high oxophilicity of high-valence iron. This model is supported by catalytic runs in the presence of diphenylamine as an oxygen radical trap aimed at probing the formation of $\mathrm{tBuOO}^{\circ}$ or $\mathrm{tBuO}^{\circ}$ radicals (entry 11). ${ }^{21,35}$ Under these conditions, product yield dropped to $<3 \%$ after $24 \mathrm{~h}$, indicating the relevance of oxygen radicals for enabling catalytic turnover and hence indirectly supporting a mechanistic model involving the intermediates with $\mathrm{Fe}$ in spin-unpaired higher oxidation states.

Since the ortho-PYA pincer iron complex 7 showed the best results throughout, further optimization of the catalytic reaction was performed with this complex. Increasing the reaction temperature to $80{ }^{\circ} \mathrm{C}$ resulted in a higher reaction rate and a 91\% product yield within $2 \mathrm{~h}$. However, full conversion was not reached due to the consumption of the oxidant as observed by the ${ }^{1} \mathrm{H}$ NMR analysis. Adjustment of the protocol therefore included batchwise additions of 1 equiv. TBHP at the onset and again after $30 \mathrm{~min}$. This portioned addition afforded $98 \%$ conversion after only $1 \mathrm{~h}$ using $0.5 \mathrm{~mol} \%$ of complex 7 (entry 12), compared to a 50\% yield with 1 mol\% $\mathrm{FeCl}_{2}$ (entry 13 ), and $<5 \%$ with only the ligand or blank reactions (entries 14, 15, entries S1-S8 in Table S2 $\dagger$ ). The catalytic species shows excellent stability. A catalyst loading as low as $0.005 \mathrm{~mol} \%$ gave a $92 \%$ yield after $24 \mathrm{~h}$ and full conversion after $48 \mathrm{~h}$, resulting in 20000 TONs (entry 16). ${ }^{43}$ Such high TONs are unusual for iron complexes and are assumed to be a direct consequence of the integrity of the catalytic species due to the rigid tridentate coordination of the PYA pincer ligand combined with the beneficial electronic impact of the ONO donor motif.

The scope of the bis-pincer iron complex 7 in oxidative catalysis was explored with a small variety of distinct substrates (Table 2). ${ }^{44}$ Diphenylmethanol was oxidized quantitatively to benzophenone, indicating that sterically more bulky substrates are converted equally well as 1-phenylethanol (entry 1). In contrast, primary alcohols are not selectively transformed to the corresponding aldehyde. For example, benzyl alcohol produces benzaldehyde in reasonable selectivity initially (32\% conversion, $78 \%$ selectivity to benzaldehyde after $2 \mathrm{~h}$, entry 2 ) and longer reaction times led to full conversion to a mixture of products, however, with only traces of benzaldehyde. Aliphatic alcohols such as cyclohexanol and 4-phenyl-2-butanol were also converted, though yields were only moderate $(75 \%$ and $53 \%$, respectively) and did not improve upon prolonging the reaction time beyond $5 \mathrm{~h}$ (entries 3,4). Thioethers such as thioanisole were oxidized with high selectivity towards the sulfoxide product with only minute overoxidation to the corresponding sulfone product even after extended reaction periods (98/2 ratio after $20 \mathrm{~h}$, entry 5$)$. Notably, oxidation of pyridine to its $\mathrm{N}$-oxide does not proceed (entry 6), indicating selective oxygen transfer with complex 7 to sulfur but not to nitrogen. This selectivity was further evaluated in the oxidation of 1-(2pyridyl)ethanol, which afforded exclusively and quantitatively
Table 2 Catalytic oxidation with $7^{a}$

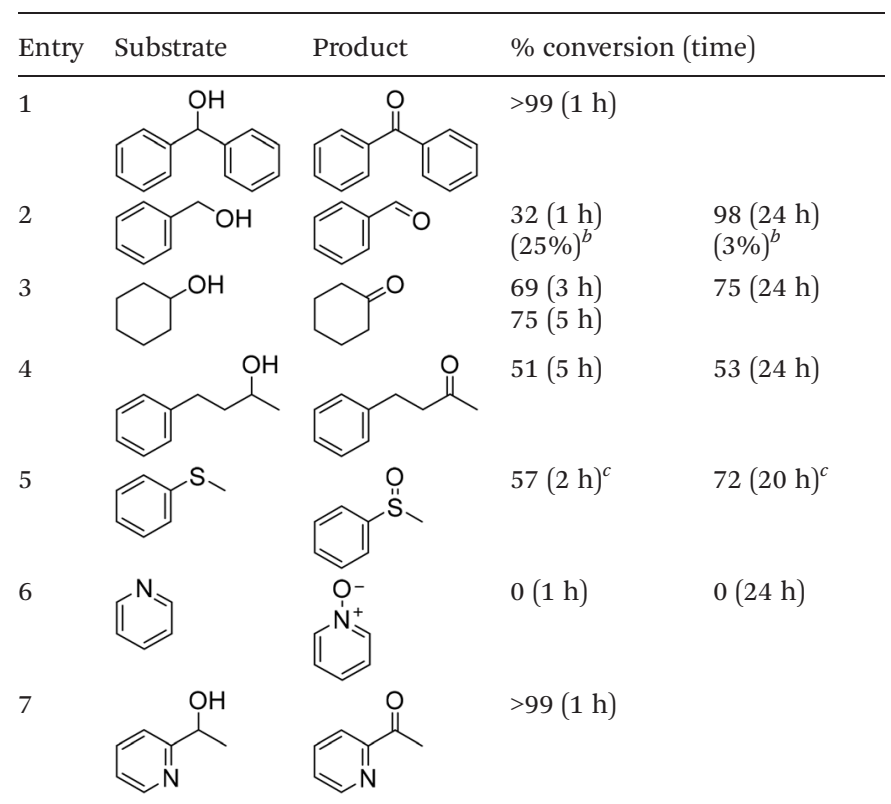

${ }^{a}$ General conditions: iron complex (3 $\mu \mathrm{mol}, 0.5 \mathrm{~mol} \%$ ), MeCN $(0.5 \mathrm{~mL})$, substrate $(0.7 \mathrm{mmol}), 1$ eq. TBHP $(0.7 \mathrm{mmol}, 5 \mathrm{M}$ in decane $)$ and a second eq. TBHP after $30 \mathrm{~min}$, $\mathrm{Ar}$ atmosphere, conversions determined by GC analysis or ${ }^{1} \mathrm{H}$ NMR spectroscopy. ${ }^{b}$ Spectroscopic yield of benzaldehyde. ${ }^{c}$ Sulfoxide/sulfone ratio 98:2 independent of reaction time.

2-acetylpyridine, demonstrating high preference for alcohol over nitrogen oxidation and no catalyst inhibition by the pyridine functionality (entry 7 ).

\section{Conclusions}

In summary, we present the synthesis of the first iron-based PYA complexes having an $\mathrm{NN}(\mathrm{N})$ or ONO coordination. The molecular structures reveal the connection between resonance structures and binding mode: the $\mathrm{NN}(\mathrm{N})$ coordinating structures have an increased resonance contribution of the diene form, while the zwitterionic resonance structure with a negative charge on the oxygen center is predominant in the ONO complexes. Peroxide-mediated alcohol oxidation provided a useful probe for the integrity of the complexes. While NN-bidentate coordination is too unstable and leads to complex decomposition, NNN-tridentate binding results in more rigid iron coordination, yet also to catalytic inhibition. In contrast, ONO-tridentate PYA pincer coordination has a positive effect on the stability of the iron complex and its catalytic activity, and this ligand imparts high product yields and excellent TONs. Catalytic oxidation additionally proceeds with sterically more demanding substrates, aliphatic alcohols, and in oxygen transfer oxidation to form sulfoxide, yet leaves imines unaffected. This work highlights the unique coordination flexibility of PYA ligands and their potential to induce attractive catalytic performance with Earth-abundant 
first row transition metals. Future work will focus on extending the catalytic scope of these iron complexes to other oxidative transformations.

\section{Experimental section}

\section{General considerations}

All reagents were commercially available and used as received unless specified differently. When specified, reactions were run in an MBRAUN LABmaster 130 argon-filled glovebox. When using inert conditions, the chemicals and materials were dried and degassed prior to use. THF, MeCN, hexanes, and $\mathrm{Et}_{2} \mathrm{O}$ were taken from a solvent purification system (SPS), stored over molecular sieves for at least 2 days, degassed with $\mathrm{N}_{2}$ gas bubbling for $30 \mathrm{~min}$, placed in the glovebox and stored over fresh molecular sieves prior to use. $\mathrm{FeCl}_{2}, \mathrm{ZnCl}_{2}, \mathrm{NEt}_{4} \mathrm{I}$, and $\mathrm{Na}_{2} \mathrm{CO}_{3}$ were dried under vacuum for 1-2 days before placing them in the glovebox. Mesitylene and 1-phenyl ethanol were placed on molecular sieves in a Schlenk flask, degassed with $\mathrm{N}_{2}$ bubbling for $30 \mathrm{~min}$, and used under inert $\mathrm{N}_{2}$ conditions. $\mathrm{CD}_{3} \mathrm{CN}$ and DMSO- $d_{6}$ were placed in the glovebox directly, and dried further over molecular sieves. Compounds

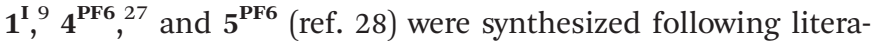
ture procedures. ${ }^{1} \mathrm{H},{ }^{13} \mathrm{C},{ }^{19} \mathrm{~F}$, and ${ }^{31} \mathrm{P}$ NMR spectra were recorded at $25{ }^{\circ} \mathrm{C}$ using a Bruker spectrometer operating at 300, 75, 282, and $121 \mathrm{MHz}$, respectively. ${ }^{1} \mathrm{H}$ and ${ }^{13} \mathrm{C}$ NMR chemical shifts are reported in ppm relative to $\mathrm{SiMe}_{4}$ by using the residual solvent resonance as the internal standard. ${ }^{31} \mathrm{P}$ NMR chemical shifts are externally referenced to $85 \%$ aqueous $\mathrm{H}_{3} \mathrm{PO}_{4} \cdot{ }^{19} \mathrm{~F}$ NMR chemical shifts are externally referenced to $\mathrm{CFCl}_{3}$. The purity of the bulk samples of the complexes has been established by NMR spectroscopy, and when possible by elemental analysis. The elemental analyses were performed at the Department of Chemistry and Biochemistry (DCB), University of Bern, Microanalytic Laboratory using a Thermo Scientific Flash 2000 CHNS-O elemental analyzer. High-resolution mass spectrometry was carried out using a Thermo Scientific LTQ Orbitrap XL (ESI-TOF) by the mass spectroscopy group of the DCB. Cyclic voltammograms were recorded using an Autolab PGSTAT101 from Metrohm in the specified solvent solutions: $10 \mathrm{~mL}$ solvent, $1 \mathrm{mM}$ sample, and $100 \mathrm{mM}$ tetrabutylammonium hexafluorophosphate $\left(\mathrm{nBu}_{4} \mathrm{~N}\right) \mathrm{PF}_{6}$ as supporting electrolyte. Solutions were taken under argon from the glovebox. Redox potentials were measured using a glassy carbon working electrode, $\mathrm{Ag} / \mathrm{AgCl}$ reference electrode (SSCE) and Ptwire auxiliary electrode and are tabulated versus a ferrocene internal standard. ${ }^{45}$ IR spectra were recorded using a Jasco FT/ IR 4700 Fourier transform infrared spectrometer.

\section{Syntheses}

$\mathbf{1}^{\text {PF6 }}$. In a round bottom flask in air, $\mathbf{1}^{\mathbf{I}}(404 \mathrm{mg}, 1.18 \mathrm{mmol})$ was dissolved in water $(35 \mathrm{~mL})$ to which a solution of $\mathrm{NH}_{4} \mathrm{PF}_{6}$ (600 mg, $3.7 \mathrm{mmol})$ in demi water $(10 \mathrm{~mL})$ was added while stirring. The clear colorless solution turned to a white suspension and was stirred for $10 \mathrm{~min}$. The solid was collected by fil- tration, washed with demi water $(2 \times 5 \mathrm{~mL})$ and $\mathrm{Et}_{2} \mathrm{O}(2 \times$ $10 \mathrm{~mL}$ ) and dried in a vacuum, resulting in $\mathbf{1}^{\mathbf{P F} 6}$ as a slightly off-white powder $(87 \%, 370 \mathrm{mg}) .{ }^{1} \mathrm{H}$ NMR (DMSO- $\left.d_{6}\right): \delta_{\mathrm{H}} 12.03$ $(\mathrm{s}, 1 \mathrm{H}, \mathrm{NH}), 8.84$ (d, $J=4 \mathrm{~Hz}, 1 \mathrm{H}, \mathrm{Ar}-H), 8.80(\mathrm{~d}, J=7 \mathrm{~Hz}, 2 \mathrm{H}$, $\operatorname{Ar}-H$ ), 8.51 (d, $J=7 \mathrm{~Hz}, 2 \mathrm{H}, \operatorname{Ar}-H), 8.25$ (d, $J=8 \mathrm{~Hz}, 1 \mathrm{H}, \operatorname{Ar}-H$ ), $8.16(\mathrm{td}, J=8 \mathrm{~Hz}, J=1 \mathrm{~Hz}, 1 \mathrm{H}, \mathrm{Ar}-H), 7.80(\mathrm{~m}, 1 \mathrm{H}, \mathrm{Ar}-H), 4.20$ $\left(\mathrm{s}, 3 \mathrm{H},-\mathrm{CH}_{3}\right) \cdot{ }^{19} \mathrm{~F}$ NMR (DMSO- $\left.d_{6}\right): \delta_{\mathrm{F}}-70.12\left(\mathrm{~d}, J_{\mathrm{FP}}=705 \mathrm{~Hz}\right.$, 1F). ${ }^{31} \mathrm{P}$ NMR (DMSO- $d_{6}$ ): $\delta_{\mathrm{P}}-144.2\left(\mathrm{~s},{ }^{2} \mathrm{JPF}_{\mathrm{PF}}=710 \mathrm{~Hz}, \mathrm{PF}_{6}\right) \cdot{ }^{13} \mathrm{C}$ NMR (DMSO- $\left.d_{6}\right): \delta_{\mathrm{C}} 164.80(C \mathrm{q}), 151.47(C \mathrm{q}), 148.87(\mathrm{CH})$, $148.24(\mathrm{Cq}), 145.94(\mathrm{CH}), 138.61(\mathrm{CH}), 128.31(\mathrm{CH}), 123.59$ $(\mathrm{CH}), 115.91(\mathrm{CH}), 46.49\left(\mathrm{CH}_{3}\right) . \mathrm{IR}\left(\mathrm{cm}^{-1}\right): 3352,3103,1719$, $1637,1583,1515,1460,1433,1282,1253,1154,1113,1031$, 1002. ESI-MS $m / z$ 214.0973, calc: 214.0975.

$\left[\mathrm{Fe}(\text { pyPYA }) \mathrm{Cl}_{2}\right]_{2}$ (2). In the glovebox in a $15 \mathrm{~mL}$ vial, ${ }_{1}^{\text {PF6 }}$ (100 $\mathrm{mg}, 0.30 \mathrm{mmol}$ ) was dissolved in THF $(1 \mathrm{~mL})$ to which a solution of LiHMDS (48 mg, $0.29 \mathrm{mmol})$ in THF (3 mL) was added, resulting in an instant color change from orange to dark red. The mixture was stirred for $15 \mathrm{~min}$, after which a solution of $\mathrm{FeCl}_{2}$ (36 mg, $0.29 \mathrm{mmol}$ ) in THF (4 mL) was added. The resulting dark red suspension was stirred for $16 \mathrm{~h}$ and the solid was collected by filtration. The product was washed with $\mathrm{Et}_{2} \mathrm{O}(2 \times 3 \mathrm{~mL})$ and hexanes $(3 \mathrm{~mL})$ and dried in a vacuum to afford 2 as a dark red highly air-sensitive powder (84 mg, 86\%). ${ }^{1} \mathrm{H}$ NMR $\left(\mathrm{CD}_{3} \mathrm{CN}\right): \delta_{\mathrm{H}} 73.13,64.25$ (minor), $54.51,9.88,8.04,4.02,0.02,-15.83,-50.21$. IR $\left(\mathrm{cm}^{-1}\right): 3052$, 1646, 1589, 1499, 1334, 1249, 1200, 1128, 1046, 1019, 912, 839, $755,695,556,468$. Magnetic susceptibility following the Evans method was performed in a 50:1 mixture of DMSO- $d_{6} / \mathrm{DMSO}$, resulting in a $\mu_{\text {eff: }}: 4.9, S=2$. Due to the high sensitivity of the compound, no satisfactory elemental analysis was performed.

$\left[\mathbf{F e}(\right.$ pyPYA $\left.) \mathbf{C l}(\mathbf{P y})_{3}\right] \mathbf{I}(3)$. In the glovebox in a $15 \mathrm{~mL}$ vial, $\mathbf{1}^{\mathbf{I}}$ (120 mg, $0.35 \mathrm{mmol}$ ) was dissolved in THF $(2 \mathrm{~mL})$ to which LiHMDS (59 mg, $0.35 \mathrm{mmol}$ ) was added, resulting in an instant color change to dark red. The mixture was stirred for $1 \mathrm{~h}$ and a solution of $\mathrm{FeCl}_{2}(45 \mathrm{mg}, 0.35 \mathrm{mmol})$ in THF $(2 \mathrm{~mL})$ was subsequently added. The resulting dark red suspension was stirred overnight, filtered, and the solids were washed with $\mathrm{Et}_{2} \mathrm{O}(2 \times 3 \mathrm{~mL})$ and hexanes $(3 \mathrm{~mL})$ and dried in a vacuum, resulting in a brown powder. A part of the product $(19.8 \mathrm{mg}$, $0.03 \mathrm{mmol})$ was suspended in THF $(1 \mathrm{~mL}) . \mathrm{NEt}_{4} \mathrm{I}(15.4 \mathrm{mg}$, $0.06 \mathrm{mmol})$ in THF $(3 \mathrm{~mL})$ was added and the mixture was stirred for $30 \mathrm{~min}$ before pyridine $(1 \mathrm{~mL})$ was added. The resulting suspension was stirred for $16 \mathrm{~h}$. The solid was removed by filtration and the filtrate was crystallized by layering of hexanes over 5 days. The formed crystalline material was collected, washed with $\mathrm{Et}_{2} \mathrm{O}(3 \times 2 \mathrm{~mL})$ and left to dry overnight (applying vacuum leads to product decomposition), resulting in the product as a yellow-to-brown powder in $21 \%$ yield (4.2 mg, $6.3 \mu \mathrm{mol}) .{ }^{1} \mathrm{H}$ NMR (THF/pyridine, crude reaction mixture): $\delta_{\mathrm{H}} 69.09$ (minor), 58.23, 52.37 (minor), 46.58, 34.17. NMR analysis of the crystalline product is not possible due to instability upon redissolution. IR $\left(\mathrm{cm}^{-1}\right)$ : 3052, 2360, 2028, 1640, 1595, 1572, 1527, 1499, 1440, 1385, 1291, 1200, 1145, 1067, 1034, 1006, 891, 812, 755, 698, 522, 485, 456, 421. The quantities were too low and the compound too air-sensitive for elemental analysis. 
$\left[\mathrm{Fe}\left(\mathbf{\kappa}^{\mathrm{NNN}}-\mathrm{pyPYA}_{2}\right) \mathrm{Cl}_{2}\right]$ (6). In the glovebox in a $15 \mathrm{~mL}$ vial, $4^{\mathrm{PF} 6}(100 \mathrm{mg}, 0.156 \mathrm{mmol})$ and $\mathrm{Na}_{2} \mathrm{CO}_{3}(50 \mathrm{mg}, 0.479 \mathrm{mmol})$ were suspended in anhydrous DMF $(3 \mathrm{~mL})$. The mixture was stirred for $1 \mathrm{~h}$, after which it was filtered into a solution of $\mathrm{FeCl}_{2}(19.8 \mathrm{mg}, 0.156 \mathrm{mmol})$ in anhydrous DMF $(2 \mathrm{~mL})$, resulting in a direct color change from orange to intense purple. The mixture was stirred at room temperature for $16 \mathrm{~h}$, after which it was placed at $-50{ }^{\circ} \mathrm{C}$ for $1 \mathrm{~h}$. The solution was removed by decantation, and the solids were washed with $\mathrm{Et}_{2} \mathrm{O}$ $(3 \times 4 \mathrm{~mL})$ and dried in a vacuum to yield the title product as an intense purple solid $\left(63 \%, 46.3 \mathrm{mg}\right.$ ). ${ }^{1} \mathrm{H}$ NMR (DMSO- $d_{6}$ ): $\delta_{\mathrm{H}} 73.02,18.42,10.88 . \mathrm{IR}\left(\mathrm{cm}^{-1}\right): 3041,1647,1607,1582,1501$, 1360, 1334, 1242, 1201, 1133, 1071, 1031, 940, 847, 765, 689, 524, 446. ESI-MS $\mathrm{m} / \mathrm{z}$ 473.0119, calc: 473.0103. Elem. anal. calcd for $\mathrm{C}_{19} \mathrm{H}_{17} \mathrm{Cl}_{2} \mathrm{FeN}_{5} \mathrm{O}_{2} \times 1.5 \mathrm{H}_{2} \mathrm{O}: \mathrm{C}$ 45.54, $\mathrm{H}$ 4.02, $\mathrm{N}$ $13.97 \%$; found: C $45.82, \mathrm{H} 3.51, \mathrm{~N} 13.88 \%$. Magnetic susceptibility following the Evans method was performed in a $95: 5$ mixture of DMSO- $d_{6} / \mathrm{DMSO}$, resulting in a $\mu_{\text {eff }}: 4.9, S=2$.

$\left[\mathrm{Fe}\left(\mathrm{pyPYA}_{2}\right)_{2}\right]\left(\mathrm{PF}_{6}\right)_{2} \quad(7)$ and $\left[\mathrm{Fe}\left(\kappa^{\mathrm{ONO}}-\mathrm{pyPYA}_{2}\right) \mathrm{Cl}_{2}\right] \quad$ (8). Analogues to the synthesis of 6 with the same amounts, using $5^{\mathbf{P F 6}}$, gave a solid which was washed with $\mathrm{MeCN}(3 \times 3 \mathrm{~mL})$ to yield the title product as a purple solid $(49 \%, 40 \mathrm{mg}) .{ }^{1} \mathrm{H}$ NMR (DMSO- $\left.d_{6}\right): \delta_{\mathrm{H}} 70.90,11.53,10.83,7.95$. IR $\left(\mathrm{cm}^{-1}\right): 3054,1628$, 1588, 1525, 1497, 1440, 1386, 1297, 1274, 1147, 1066, 952, 839, $780,756,729,685,495,427$. ESI-MS $m / z\left[\mathrm{M}_{-2} \mathrm{PF}_{6}\right]^{2+} 375.1064$, calc 375.1046. Elem. anal. calcd for $\mathrm{C}_{38} \mathrm{H}_{34} \mathrm{~F}_{12} \mathrm{FeN}_{10} \mathrm{O}_{4} \mathrm{P}_{2}$ : C 43.86, H 3.29, N 13.46\%; found: C 43.81, H 3.20, N 13.57\%. Overlaying the decantated DMF solution with $\mathrm{Et}_{2} \mathrm{O}$ afforded crystals of compound 8 in small quantities ( $<5 \mathrm{mg}$ ). ESI-MS $\mathrm{m} / \mathrm{z}[\mathrm{M}]^{+}$473.0118, calc: 473.0103; $[\mathrm{M}-\mathrm{Cl}]^{+}$438.0430, calc: 438.0415 .

\section{Representative catalytic procedures}

In an argon filled glovebox, a $5 \mathrm{~mL}$ Teflon capped microwave reaction vial was loaded with the catalyst $(1 \mathrm{~mol} \%, 7 \mu \mathrm{mol})$, closed and transferred to a fume hood. While stirring, MeCN (0.5 mL), the substrate (1-phenylethanol, $0.7 \mathrm{mmol}$ ), and oxidant (TBHP $5 \mathrm{M}$ in decane, $1.05 \mathrm{mmol}$ ) were added consecutively by (micro)syringes. The vial was placed in a heating bath. On set times, $0.05 \mathrm{~mL}$ samples were taken using a $1 \mathrm{~mL}$ syringe, diluted in $\mathrm{CD}_{3} \mathrm{CN}(0.4 \mathrm{~mL})$, filtered through a cotton pad, and analyzed by ${ }^{1} \mathrm{H}$ NMR spectroscopy. When using an NMR standard, the reaction was performed without sampling. Mesitylene (0.5 eq.) was added at the end of the reaction to avoid interference, and a sample was taken and analyzed by NMR spectroscopy as described above. The amounts were adjusted as follows when using lower catalyst loadings: for $0.1 \mathrm{~mol} \%$ runs: $4 \mu \mathrm{mol}$ catalyst in $3 \mathrm{~mL} \mathrm{MeCN}$ with $4 \mathrm{mmol}$ 1-phenylethanol and $6 \mathrm{mmol}$ TBHP; for $0.005 \mathrm{~mol} \%$ run: $2 \mu \mathrm{mol}$ catalyst in $3 \mathrm{~mL}$ of MeCN with $20 \mathrm{mmol}$ 1-phenylethanol and $2 \times 20$ mmol TBHP. ${ }^{43}$

\section{Conflicts of interest}

There are no conflicts to declare.

\section{Acknowledgements}

We acknowledge generous financial support from the European Research Council (CoG 615653), the Seal of Excellence Fund of the University of Bern (SELF fellowship to D. G. A. V.), and the Swiss National Science Foundation (200020_182663). We thank Fabio Notter for synthetic assistance and the group of Chemical Crystallography of the University of Bern for X-ray analysis of all reported structures.

\section{Notes and references}

1 A. Togni and L. M. Venanzi, Nitrogen Donors in Organometallic Chemistry and Homogeneous Catalysis, Angew. Chem., Int. Ed. Engl., 1994, 33, 497-526.

2 P. D. W. Boyd, L. J. Wright and M. Zafar, Extending the Range of Neutral N-Donor Ligands Available for Metal Catalysts: $\quad N$-[1-Alkylpyridin-4(1H)-ylidene]amides in Palladium-Catalyzed Cross-Coupling Reactions, Inorg. Chem., 2011, 50, 10522-10524.

3 M. E. Doster and S. A. Johnson, Selective C-F Bond Activation of Tetrafluorobenzenes by Nickel(0) with a Nitrogen Donor Analogous to $N$-Heterocyclic Carbenes, Angew. Chem., Int. Ed., 2009, 48, 2185-2187.

4 Q. Shi, R. J. Thatcher, J. Slattery, P. S. Sauari, A. C. Whitwood, P. C. McGowan and R. E. Douthwaite, Synthesis, Coordination Chemistry and Bonding of Strong $N$-Donor Ligands Incorporating the $1 \mathrm{H}$-Pyridin- $(2 E)$-Ylidene (PYE) Motif, Chem. - Eur. J., 2009, 15, 11346-11360.

5 R. J. Thatcher, D. G. Johnson, J. M. Slattery and R. E. Douthwaite, Charged Behaviour from Neutral Ligands: Synthesis and Properties of $\mathrm{N}$-Heterocyclic Pseudo-amides, Chem. - Eur. J., 2012, 18, 4329-4336.

6 V. Leigh, D. J. Carleton, J. Olguin, H. Mueller-Bunz, L. J. Wright and M. Albrecht, Solvent-Dependent Switch of Ligand Donor Ability and Catalytic Activity of Ruthenium(II) Complexes Containing Pyridinylidene Amide (PYA) N-Heterocyclic Carbene Hybrid Ligands, Inorg. Chem., 2014, 53, 8054-8060.

7 C.-H. Chien, M.-K. Leung, J.-K. Su, G.-H. Li, Y.-H. Liu and Y. Wang, Substituent Effects on Pyrid-2-yl Ureas toward Intramolecular Hydrogen Bonding and Cytosine Complexation, J. Org. Chem., 2004, 69, 1866-1871.

8 K. F. Donnelly, C. Segarra, L.-X. Shao, R. Suen, H. MüllerBunz and M. Albrecht, Adaptive $N$-Mesoionic Ligands Anchored to a Triazolylidene for Ruthenium-Mediated (De) Hydrogenation Catalysis, Organometallics, 2015, 34, 40764084.

9 M. Navarro, V. Rosar, T. Montini, B. Milani and M. Albrecht, Olefin Dimerization and Isomerization Catalyzed by Pyridylidene Amide Palladium Complexes, Organometallics, 2018, 37, 3619-3630.

10 . DFT computational investigation to NN vs. NO binding: C. M. A. Muller, M. V. Babak, M. Kubanik, M. Hanif, S. M. F. Jamieson, A. G. Hartinger and L. J. Wright, Pt(II) 
pyridinium amidate (PYA) complexes: Preparation and in vitro anticancer activity studies, Inorg. Chim. Acta, 2016, 450, 124-130.

11 (a) O. Belda and C. Moberg, Bispyridylamides-coordination chemistry and applications in catalytic reactions, Coord. Chem. Rev., 2005, 249, 727-740; (b) A. Rajput and R. Mukherjee, Coordination chemistry with pyridine/pyrazine amide ligands. Some noteworthy results, Coord. Chem. Rev. , 2013, 257, 350-368.

12 M. Navarro, M. Li, H. Müller-Bunz, S. Bernhard and M. Albrecht, Donor-Flexible Nitrogen Ligands for Efficient Iridium-Catalyzed Water Oxidation Catalysis, Chem. - Eur. J., 2016, 22, 6740-6745.

13 M. Navarro, C. A. Smith, M. Li, S. Bernhard and M. Albrecht, Optimization of Synthetically Versatile Pyridylidene Amide Ligands for Efficient Iridium-Catalyzed Water Oxidation, Chem. - Eur. J., 2018, 24, 6386-6398.

14 K. Salzmann, C. Segarra and M. Albrecht, Donor-flexible bis-pyridine amide ligands for highly efficient rutheniumcatalyzed olefin oxidation, Angew. Chem., Int. Ed., 2020, 59, 8932-8936.

15 (a) M. S. Chen and M. C. A. White, Predictably Selective Aliphatic C-H Oxidation Reaction for Complex Molecule Synthesis, Science, 2007, 318, 783-787; (b) M. Cianfanelli, G. Olivio, M. Milan, R. J. M. Klein Gebbink, X. Ribas, M. Bietti and M. Costas, Enantioselective $\mathrm{C}-\mathrm{H}$ Lactonization of Unactivated Methylenes Directed by Carboxylic Acids, J. Am. Chem. Soc., 2020, 142, 1584-1593.

16 C. Kim, K. Chen, J. Kim and L. Que Jr., Stereospecific Alkane Hydroxylation with $\mathrm{H}_{2} \mathrm{O}_{2}$ Catalyzed by an Iron(II)Tris(2-pyridylmethyl)amine Complex, J. Am. Chem. Soc., 1997, 119, 5964-5965.

17 P. Talsi and K. P. Bryliakov, Chemo- and stereoselective C$\mathrm{H}$ oxidations and epoxidations/cis-dihydroxylations with $\mathrm{H}_{2} \mathrm{O}_{2}$, catalyzed by non-heme iron and manganese complexes, Coord. Chem. Rev., 2012, 256, 1418-1434.

18 Z. Guan, Metal Catalysts for Olefin Polymerization, Top Organomet Chem., Springer-Verlag Berlin Heidelberg, 2009, vol. 26, ISBN 978-3-540-87750-9.

19 G. J. P. Britovsek, V. C. Gibson, B. S. Kimberley, P. J. Maddox, S. J. McTavish, G. A. Solan, A. J. P. White and D. J. Williams, Novel olefin polymerization catalysts based on iron and cobalt, Chem. Commun., 1998, 849-850.

20 M. W. Bouwkamp, E. Lobkovsky and P. J. Chirik, Bis(imino) pyridine Iron(II) Alkyl Cations for Olefin Polymerization, J. Am. Chem. Soc., 2005, 127, 9660-9661.

21 A. Gonzalez-de-Castro and J. Xiao, Green and Efficient: Iron-Catalyzed Selective Oxidation of Olefins to Carbonyls with $\mathrm{O}_{2}$, J. Am. Chem. Soc., 2015, 137, 8206-8218.

22 . The solution state magnetic moment of 2 was determined per iron center, indicating that the dimeric complex either dissociates to monomeric $\mathrm{Fe}(\mathrm{L}) \mathrm{Cl}_{2}$ complexes in solution, or that the Fe centers in the dimeric complex have a very weak magnetic coupling if any at all.

23 . Crystallographic data of the complexes reported in this paper have been deposited with the Cambridge
Crystallographic Data Centre (CCDC) as supplementary publication numbers 2001555 (2), 2001556 (3), 2001557 (6), 2001558 (7). Details of data collection, structure solution, and refinement are compiled in the ESI. $\dagger$

24 . Yields were low due to crystallization of the product. The product is sensitive to vacuum, as it leads to gradual decomposition.

25 . O-coordination has been observed in neutral pyridine carboxamides without $N$-deprotonation, see: W. Jacob and R. Mukherjee, Synthesis, structure, and properties of monomeric Fe(II), Co(II), and Ni(II) complexes of neutral N(aryl)-2-pyridinecarboxamides, Inorg. Chim. Acta, 2006, 359(14), 4565-4573.

26 A. Dorazco-González, H. Höpfl, F. Medrano and A. K. Yatsimirsky, Recognition of Anions and Neutral Guests by Dicationic Pyridine-2,6-dicarboxamide Receptors, J. Org. Chem., 2010, 75, 2259-2273.

27 (a) P. Melle, Y. Manoharan and M. Albrecht, Modular Pincer-type Pyridylidene Amide Ruthenium(II) Complexes for Efficient Transfer Hydrogenation Catalysis, Inorg. Chem., 2018, 57, 11761-11774; (b) P. Melle and M. Albrecht, Ruthenium Complexes with PYA Pincer Ligands for Catalytic Transfer Hydrogenation of Challenging Substrates, Chimia, 2019, 73, 299-303.

28 P. Melle, N. Segaud and M. Albrecht, Ambidentate bonding and electrochemical implications of pincer-type pyridylidene amide ligands in complexes of nickel, cobalt and zinc, Dalton Trans., 2020, 49, 12662-12673.

29. Due to low solubility of 6 in MeCN, and reactivity of 7 with DMF, the complexes could not be analyzed in the same solvent, which limits a direct comparison of the oxidation potentials.

30 . Low solubility of the complex hampered the accurate measurement of its magnetic susceptibility by Evans method.

31 . This shielding seems to be more relevant for first-row transition metals with small ionic radii, since larger metal ions such as Ir(III), Ru(II), and Pd(II) were previously shown to coordinate to the amide nitrogen also in ortho-PYA complexes, see ref. 9, 12 and 28, while amide O-coordination was shown with Co and Ni, see ref. 28.

32 M. Ray, D. Ghosh, Z. Shirin and R. Mukherjee, Highly Stabilized Low-Spin Iron(III) and Cobalt(III) Complexes of a Tridentate Bis-Amide Ligand 2,6-Bis( $N$-phenylcarbamoyl) pyridine. Novel Nonmacrocyclic Tetraamido-N Coordination and Two Unusually Short Metal-Pyridine Bonds, Inorg. Chem., 1997, 36, 3568-3572.

33 (a) G. L. Guillet, J. B. Gordon, G. N. Di Francesco, M. W. Calkins, E. Čižmár, K. A. Abboud, M. W. Meisel, R. García-Serres and L. J. Murray, A Family of Tri- and Dimetallic Pyridine Dicarboxamide Cryptates: Unusual O, N,O-Coordination and Facile Access to Secondary Coordination Sphere Hydrogen Bonding Interactions, Inorg. Chem., 2015, 54, 2691-2704; (b) . For a related example with Ru, see also: J.-C. Waslike, G. Wu, X. Bu, G. Kehr and G. Erker, Ruthenium carbene Complexes 
Featuring a Tridentate Pincer-Type Ligand, Organometallics, 2005, 17, 4289-4297.

34 T. C. Harrop, L. A. Tyler, M. M. Olmstead and P. K. Mascharak, Change in the Spin State of an $\mathrm{Fe}^{\mathrm{III}}$ Center upon One N-to-O Switch in the Coordination of a 2,6-Pyridinedicarboxamido Unit: The Effect of Methyl Thioether and Methyl Ether Appendages at the Ligand Periphery, Eur. J. Inorg. Chem., 2003, 475-481.

35 M. F. Pinto, B. d. P. Cardoso, S. Barroso, A. M. Martins and B. Royo, Chelating bis-N-heterocyclic carbene complexes of iron(II) containing bipyridyl ligands as catalyst precursors for oxidation of alcohols, Dalton Trans., 2016, 45, 1354113546.

36 E. Balogh-Hergovich and G. Speier, Catalytic oxidation of alcohols to carbonyl compounds with hydrogen peroxide using dinuclear iron complexes, J. Mol. Catal. A: Chem., 2005, 230, 79-83.

37 S. Tanaka, Y. Kon, T. Nakashima and K. Sato, Chemoselective hydrogen peroxide oxidation of allylic and benzylic alcohols under mild reaction conditions catalyzed by simple iron-picolinate complexes, RSC Adv., 2014, 4, 37674-37678.

38 M. Sutradhar, T. Roy Barman, A. J. L. Pombeiro and L. M. D. R. S. Martins, Cu(II) and Fe(III) Complexes Derived from N-Acetylpyrazine-2-Carbohydrazide as Efficient Catalysts Towards Neat Microwave Assisted Oxidation of Alcohols, Catalysts, 2019, 9, 1053.

39 M. Lenze and E. B. Bauer, Chemoselective, iron(II)-catalyzed oxidation of a variety of secondary alcohols over primary alcohols utilizing $\mathrm{H}_{2} \mathrm{O}_{2}$ as the oxidant, Chem. Commun., 2013, 49, 5889-5891.

40 . While 7 is only sparingly soluble in DMF, the purple suspension turns to a bright orange homogeneous solution upon addition of the oxidant.

41. The reactivity of 7 is related to the $\mathrm{Fe}(\mathrm{L}) \mathrm{Cl}_{2}$ complex reported by Ghosh and coworkers, although the catalysis reported herein vastly exceeds the earlier reported alcohol oxidation activity. N. Tyagi, O. Singh and K. Ghosh, Nonheme iron(III) complex with tridentate ligand: Synthesis, structures and catalytic oxidations of alkanes, Catal. Commun., 2017, 95, 83-87.

42 . Complex 7 reached full conversion after 9 days at $0.05 \mathrm{~mol} \%$ catalyst loading while $0.1 \mathrm{~mol} \% \mathrm{FeCl}_{2}, 2$ and 6 reached $37 \%, 43 \%$ and $23 \%$ conversion, respectively after the same time. These data underline the high activity of complex 7, although deterioration of the oxidant and background oxidations due to oxygen from the atmosphere cannot be excluded in these long-term experiments.

43 . Note that in the run with 0.005 mol\% catalyst the major solvent is decane and the minor solvent is MeCN (ratio of $8: 3$ ), yet the reaction still goes to completion in this apolar solvent mixture.

44 S.-I. Murahashi and N. Komiya, New types of catalytic oxidations in organic synthesis, Catal. Today, 1998, 41, 339349.

45 N. G. Connelly and W. E. Geiger, Chemical Redox Agents for Organometallic Chemistry, Chem. Rev., 1996, 96, 877910 . 